\title{
General Analysis of Resonance Coupled Wireless Power Transfer (WPT) Using Inductive Coils
}

\author{
Chandrasekharan NATARAJ, Sheroz KHAN, Mohammed Hadi HABAEBI, Asan G. A. MUTHALIF
}

\begin{abstract}
In this paper, parameter analysis of the inductive coils is evaluated for low power Wireless Power Transfer (WPT) applications. Inductive coils are the major element used in the WPT systems, in which different shaped coils are employed. The selection of coils is very critical, depends purely on the fundamental characteristics (shape and geometry) of the coils. In order to design a better system, three different shapes of coils, namely, circular, square and rectangular are designed and analysed. The vital parameters such as self-inductance, mutual inductance, quality factor, magnetic field and efficiency are evaluated for all three coils. It is observed that these parameters are maximal for circular as compared to the other two shapes. The circular coils produce higher voltage efficiency of $29 \%$ as compared to rectangular (25\%) and square $(23 \%)$ shaped coils. Thus, this paves a way to other researchers to suitably select circular inductive coils for wireless electricity applications.
\end{abstract}

Keywords: geometry and shape of coils; quality factor; resonant inductive coupling; self and mutual inductance; wireless power transfer

\section{INTRODUCTION}

Nikolas Tesla [1] for the first time perceives a method of inductively coupled Wireless Power Transfer (WPT). Originally, an ambitious concept, it is now an emerging source of powering current portable digital world through non-contact means. WPT is a simple and compact concept that works without wires in a near field magnetically coupled zone. The basic principle is to use two coils in appropriate orientation and maximized to its peak at resonant frequency. Such coils could be able to transfer maximum power to remote devices located centimeters range apart, a phenomenon called strongly coupled resonance mode $[2,3]$. Capacitive coupling method has also been used in the wireless applications but it is not user-friendly [4] like inductive coupling. The gap maintained between two self-resonant coils is very small, referred to as non-radiative mid-range energy transfer [5, 6]. The concept is well researched in circuit theory, however many equivalent models are being suggested and orientation for effective and maximum midrange power transfer is among the ongoing topics [7]. The applications in view are such as biomedical implants, portable devices, robots, consumer electronics, and electrical vehicles [8]. In this WPT scheme, the most significant consideration is power transfer efficiency and distance coverage both for non-oriented or properly oriented two coils. Coil geometry is one of the powerful performance key factors, which decides the distance coverage and power transfer efficiency [9]. Geometry of the coils includes the following specifications such as shape, number of turns, material and its physical parameters, inner and outer diameter of the coils, gap between the adjacent turns. Always better geometry produces good performance and high transfer efficiency. Of course, lot of studies have taken place and specified the significance of geometry. Almost all the research works address the scenario of circular coil (or spiral), which is used frequently in most of the systems [10-13]. Moreover, very few scholars have shown interest in the analysis of shapes of coils and produced results. However, there is still a requirement to conduct a detailed investigation and analysis for proving circular coil as the best suitable choice for the WPT environment. It brings great benefit for the future research works to use the different shapes of coils according to their scope and intended application.

In this work, we have carefully chosen basic RLC circuit for WPT, and used the practical values of circuit elements, and demonstrated both analytical and experimental results. The paper is organized as follows: the proposed system design model is described in Section 2 , mathematical analysis of proposed method is detailed in Section 3 and the results of both simulation and experiment works are discussed in Section 4. Finally, the conclusion is drawn in Section 5.

\section{PROPOSED SYSTEM DESIGN}

Fig. 1 illustrates the block diagram of the proposed WPT system based on inductive coupling. There are two inductive coils such as source and load coils connected in the transmitter and receiver units to design the wireless electricity system. The power is transmitted wirelessly to the receiver unit through coupled magnetic field. Then, the harvested power in the receiver circuit can energise the load, which is connected remotely with the transmitter unit.

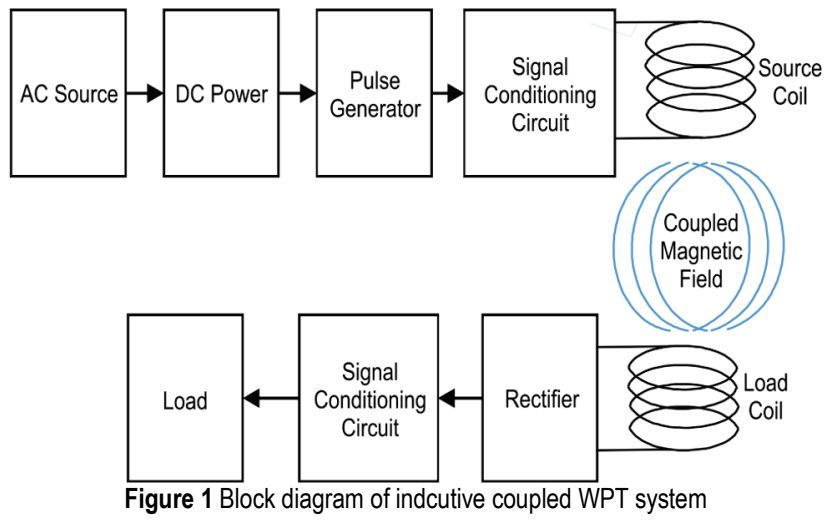

The transmitter unit consists of 555 timer based pulse generator to produce the required pulses for transmitting the power wirelessly. Initially, the input power does not contain enough strength and signal behaviour for broadcasting wirelessly. In order to strengthen the signal, 
pulse outputs from the pulse generator are passed as an input to $\mathrm{H}$ - Bridge based signal conditioning circuit. It produces the required ac signal to derive the source coil with the desired frequency.

The source coil is coupled with the load coil to produce the coupled magnetic field in the coil vicinity. It means that the applied ac source power in the form of pulses will produce fluctuating magnetic field in the coil zone. When the load coil is placed inside the coupled magnetic field, an emf will be induced across the receiver coil terminals. Then, signal conditioning circuit is used in the receiver to produce the stable dc voltage and then it is applied to power up the load. Hence, wireless power transfer is achieved from source to load coil, based on the Faraday's law of electromagnetic induction.

\section{ANALYSIS WITH MATHEMATICAL MODEL}

The electrical equivalent model of WPT system is illustrated in Fig. 2 [11]. We can consider that the coils are aligned in the centre axis irrespective of placement of coils to conduct the parameter analysis. The resistors $R_{1}$ and $R_{4}$ make up internal resistance of power source and load resistor respectively. Remaining circuit elements $R_{2}$, $R_{3}, C_{1}$, and $C_{2}$ are parasitic values of source and load coils. $L_{S}, L_{L}$ represent self-inductance of source and load coils. Similarly, $M_{S}$ and $M_{L}$ are the mutual inductances exhibited from source and load coil side respectively. The values of self and mutual inductances are geometry dependent parameters and also act as performance governing parameters of WPT system. These are variable quantities, which has been varied along with coil geometry related parameters.

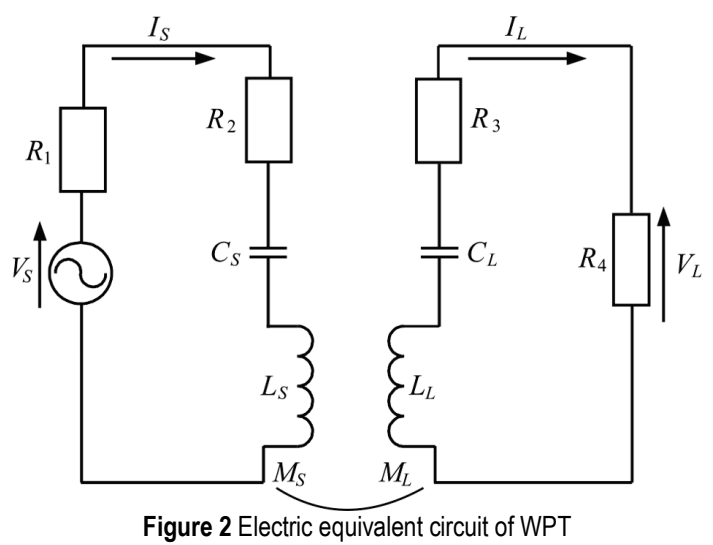

We consider the source coil with radius $r_{S}$ and number of turns $n_{S}$ in which current $I$ is applied so that it generates magnetic flux around the loop of coil by the Biot-Savart law. The magnetic field density $B$ produced away from current in $\mathrm{z}$ direction and in central axis of the coil can be expressed as [6],

$B=\frac{\mu_{i}}{4 \pi} \frac{2 n_{S} \pi r_{S}^{2}}{\left(z^{2}+r_{S}^{2}\right)^{3 / 2}}$

whereas $\mu$ and $Z$ are the permeability and distance between coils respectively. The load coil with the parameters radius $\left(r_{L}\right)$ and number of turns $\left(n_{L}\right)$ will be placed in the produced field zone, which induces voltage in the load coil through flux linkages.

The coil parameters such as self and mutual inductance of source and load coil are expressed below:

$$
\begin{aligned}
L_{S} & =\frac{\mu \pi n_{S}^{2} r_{S}}{2} \\
L_{L} & =\frac{\mu \pi n_{L}^{2} r_{L}}{2} \\
M_{S} & =\frac{\mu \pi n_{S} n_{L} r_{S}^{2} r_{L}^{2}}{2\left(z^{2}+r_{S}^{2}\right)^{3 / 2}} \\
M_{L} & =\frac{\mu \pi n_{S} n_{L} r_{S}^{2} r_{L}^{2}}{2\left(z^{2}+r_{L}^{2}\right)^{3 / 2}}
\end{aligned}
$$

From Eq. (4) and (5), it is understood that mutual inductance of both source and load coils is directly proportional to the shape of the coil and inversely proportional to the distance of coil separation ' $Z$ ' [7]. The source and load coil resonant frequencies are $\omega_{S}=1 / \sqrt{L_{S} C_{S}}$ and $\omega_{L}=1 / \sqrt{L_{L} C_{L}}$, with power transfer efficiency, $\eta$ derived in Eqs. (6) to (8).

$$
\begin{aligned}
& \eta=\frac{R_{L}\left|I_{L}\right|^{2}}{\left(R_{1}+R_{2}\right)\left|I_{S}\right|^{2}+\left(R_{3}+R_{4}\right)\left|I_{L}\right|^{2}} \\
& \eta=\frac{R_{L}\left|I_{L} / I_{S}\right|^{2}}{\left(R_{1}+R_{2}\right)+\left(R_{3}+R_{4}\right)\left|I_{L} / I_{S}\right|^{2}}
\end{aligned}
$$

Total resistance at the source and load coil can be defined as $R_{S}=R_{1}+R_{2}$ and $R_{L}=R_{3}+R_{4}$ respectively. Then, the efficiency can be written as,

$$
\eta=\frac{R_{L}\left|I_{L} / I_{S}\right|^{2}}{R_{S S}+R_{L L}\left|I_{L} / I_{S}\right|^{2}}
$$

The values of $R_{S S}, R_{L L}$ and $R_{L}$ are maintained as the circuitry constants and the variables $\left|I_{S}\right|$ and $\left|I_{L}\right|$ are the rms values of current at the source and load circuit. $I_{S}^{2} R_{S}$ and $I_{L}^{2} R_{L}$ represent the power delivered by the source and power dissipated by the load coils respectively. In Eq. (8), efficiency will be maximum, when the ratio $I_{L} / I_{S}$ is maximum with the given values of resistors.

By adopting Kirchhoff Voltage Law (KVL) in the circuit, we can find the ratio $I_{L} / I_{S}$ with coupling factor $k=M / \sqrt{L_{S} L_{L}}$ as follows:

$$
\begin{aligned}
& \left(\frac{R_{S}}{j \omega L_{S}}+1-\frac{\omega_{S}^{2}}{\omega^{2}}\right) I_{S}+k I_{L} \sqrt{\frac{L_{L}}{L_{S}}}=\frac{V_{S}}{j \omega L_{S}} \\
& k I_{S} \sqrt{\frac{L_{S}}{L_{L}}+\left(\frac{R_{L}}{j \omega L_{L}}+1-\frac{\omega_{L}^{2}}{\omega^{2}}\right) I_{L}}=0
\end{aligned}
$$


By solving the above equations we can obtain the current ratio as,

$$
\frac{I_{L}}{I_{S}}=\frac{k \sqrt{L_{S} / L_{L}}}{\frac{R_{L}}{j \omega L_{L}}+\left(1-\frac{\omega_{L}^{2}}{\omega^{2}}\right)} .
$$

Under the resonant principle, the efficiency of WPT system is maximized by applying resonant frequency,

$$
\begin{aligned}
& \omega=\frac{\sqrt{2} L_{L} \omega_{L}^{2}}{\sqrt{2 L_{L}^{2} \omega_{L}^{2}-R_{L}^{2}}} \\
& \omega \cong \omega_{L}
\end{aligned}
$$

The approximation of Eq. (13) can be deduced using coupling coefficient $(k)$ and quality factor $(Q)$.

Under the frequency condition of (13),

$$
\frac{I_{L}}{I_{S}}=-j k \frac{\omega_{L} L_{L}}{R_{L}} \sqrt{\frac{L_{S}}{L_{R}}}=-j k Q_{L} \sqrt{\frac{L_{S}}{L_{R}}}
$$

As per the current ratio shown in Eq. (14), the power transfer efficiency is significantly governed by the coil parameters such as coupling factor, quality factor, coil inductances and so on. Therefore, the efficiency of the WPT system has been enhanced by carefully choosing the coil geometrical parameters. Hence the shape and other significant parameters related with the coil geometry are the most wanted and need to be investigated further for efficient design in the WPT applications.

\section{SIMULATION AND EXPERIMENTAL RESULTS}

In order to study the suitability analysis, the relevant equations are derived and simulated in the Ansys Maxwell platform. The existing theoritical equations are adopted and used in the simulation for validating the theoritical parameters such as self inductance, mutual inductance, quality factor and magnetic field of coils. Three different coils such as spiral, rectangular and square are simulated and the respective parameters are evaluated. Then, the efficincy calculation is done through three shapes of lab-scale coils, which are wounded practically.

\subsection{Self-Inductance Test}

The self inductance of circular coil $L_{C}$ can be represented mathematically using angular integral representation [14],

$$
L_{C}=\frac{\mu N^{2}}{(b-a)^{2}} \int_{0}^{\pi} \cos \varphi \int_{a}^{b} \int_{a}^{b} \frac{y x \mathrm{~d} x \mathrm{~d} y \mathrm{~d} \varphi}{\sqrt{x^{2}+y^{2}-2 x y \cos \varphi}}
$$

where $\mathrm{a}$ and $\mathrm{b}$ are the inner and outer radius of the coil, $N$ is the total number of turns, and $\mathrm{x}, y, z$ are the coil coordinates in $3 \mathrm{D}$ coil structure.

The self inductance $L_{R}$ of rectangular coil can be expressed as [15],

$$
L_{R}=\frac{\mu_{0}}{\pi} N^{2}[P-Q]
$$

where,

$$
\begin{aligned}
& P=\left[a \times \ln \frac{2 a b}{r\left(a+\sqrt{a^{2}+b^{2}}\right)}+b \times \ln \frac{2 a b}{r\left(a+\sqrt{a^{2}+b^{2}}\right)}\right] \\
& Q=\left[2\left(a+b-\sqrt{a^{2}+b^{2}}\right)+0.25(a+b)\right]
\end{aligned}
$$

The parameter $r$ is the collective radii of the bunch of coil and it is given by $r=\frac{N \times S}{\pi}, a$ and $b$ are the length and breadth of the rectangular coil, and $\mathrm{S}$ is the cross-sectional area of copper wire. The self inductance of square coil can also be derived easily using the above rectangular coil expression (16) by making uniform length and breadth.

Table 1 Measurement of inductance as a funcion of coil thickness

\begin{tabular}{|c|c|c|c|}
\hline \multirow{2}{*}{$\begin{array}{c}\text { Thickness of } \\
\text { coil }(\mathrm{m})\end{array}$} & \multicolumn{3}{|c|}{ Inductance $(\mu \mathrm{H})$} \\
\cline { 2 - 4 } & Circular & Rectangular & Square \\
\hline 0.001 & 0.1188 & 0.1399 & 0.1416 \\
\hline 0.002 & 0.1012 & 0.0709 & 0.0726 \\
\hline 0.003 & 0.0882 & 0.0523 & 0.0545 \\
\hline 0.004 & 0.0781 & 0.0410 & 0.0427 \\
\hline 0.005 & 0.0701 & 0.0330 & 0.0347 \\
\hline 0.006 & 0.0636 & 0.0267 & 0.0284 \\
\hline 0.007 & 0.0582 & 0.0215 & 0.0232 \\
\hline 0.008 & 0.0536 & 0.0172 & 0.0189 \\
\hline 0.009 & 0.0498 & 0.0134 & 0.0151 \\
\hline 0.01 & 0.0464 & 0.0100 & 0.0117 \\
\hline
\end{tabular}

Table 2 Measurement of inductance as a function of number of turns

\begin{tabular}{|c|c|c|c|}
\hline \multirow{2}{*}{$\begin{array}{c}\text { Number of } \\
\text { turns }\end{array}$} & \multicolumn{3}{|c|}{ Inductance $(\mu \mathrm{H})$} \\
\cline { 2 - 4 } & Circular & Rectangular & Square \\
\hline 1 & 0.13 & 0.0 .122 & 0.123 \\
\hline 2 & 0.51 & 0.0 .442 & 0.449 \\
\hline 3 & 1.11 & 0.936 & 0.953 \\
\hline 4 & 1.92 & 1.590 & 1.620 \\
\hline 5 & 2.93 & 2.395 & 2.443 \\
\hline 6 & 4.11 & 3.344 & 3.412 \\
\hline 7 & 5.46 & 4.431 & 0.524 \\
\hline 8 & 6.96 & 5.651 & 5.772 \\
\hline 9 & 8.60 & 6.999 & 7.152 \\
\hline 10 & 10.38 & 8.472 & 8.662 \\
\hline
\end{tabular}

The measurement of inductance of coils is determined through the Maxwell simulation software and significant readings are noted. In order to evaluate the self inductance value, three coils are modeled using 3D simulation software with copper wire of $16 \mathrm{~cm}$ length and $1 \mathrm{~cm}$ thickness. The $16 \mathrm{~cm}$ copper wire is wounded with different number of coil turns and simulated to study the behaviour of the coils. Two different experiments such as inductance as a function of thickness and number of turns of three coils are conducted for the parameter analysis.

Tab. 1 and 2 show the simulation values of inductance as a function of coil thickness and number of turns for three different shapes of coils and its corresponding comparative plots are illustrated in Fig. 3 
and 4 respectively. It can be observed that there is an exponential rise in the inductance value for all the three shapes as the number of turns increases for a fixed length and thickness of a coil. Similarly, the self inductance of coil gets reduced when the coil thickness is increased. Hence for a given length of a wire, the circular coil has the highest value of self-inductance for both cases, thickness and number of turns.

\subsection{Mutual Inductance Test}

The phenomenon of inducing e.m.f. in the secondary coil by changing the current in the primary coil is known as mutual inductance. The mutual inductance between two coils for any shape can be calculated using the Neumann's formulae given in (19).

$M=\frac{\mu_{0} N_{1} N_{2}}{4 \pi} \int_{l_{1}}^{l_{1} l_{2}{ }^{\prime \prime}} \frac{d l d l^{\prime \prime}}{r}$

where $l_{1}$ refers to the line integral around the primary loop and $l_{2}$ refers to the line integral around the secondary loop. $N_{1}$ and $N_{2}$ are the number of turns of primary and secondary coils respectively. The distance between $d l$ and $d l^{\prime \prime}$ is denoted by $r$.

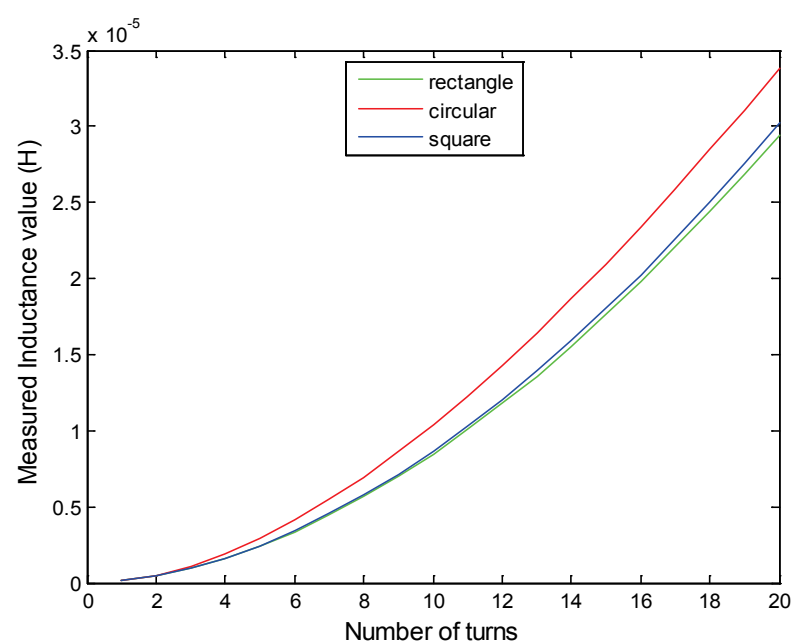

Figure 3 Measurement of inductance as a function of number of turns

The simulation of mutual inductance is carried out by fixing the distance between coils to be $3 \mathrm{~cm}$. In order to simulate the system, the three coils are modelled with desirable dimensions and a current of $5 \mathrm{~A}$ was assigned to both the source and load coils. Two different experiments such as mutual inductance as a function of thickness and number of turns are conducted for the parameter analysis. Hence for a given length of wire, a circular pair exhibits the best mutual inductance for any number of turns when compared to square and rectangular shapes as shown in Fig. 5. The highest value of mutual inductance indicates that the two coils are closely coupled and can deliver a better performance in a WPT application.

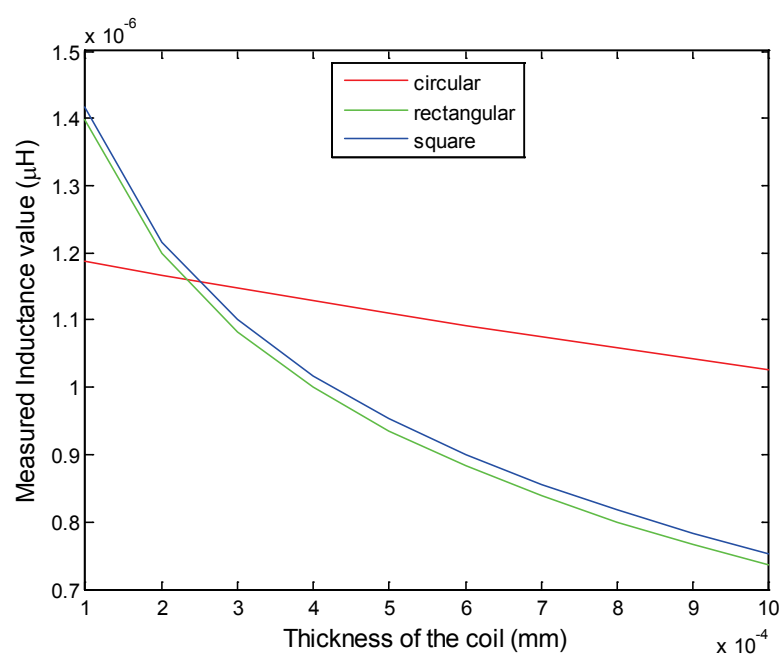

Figure 4 Measurement of inductance as a function of coil thickness

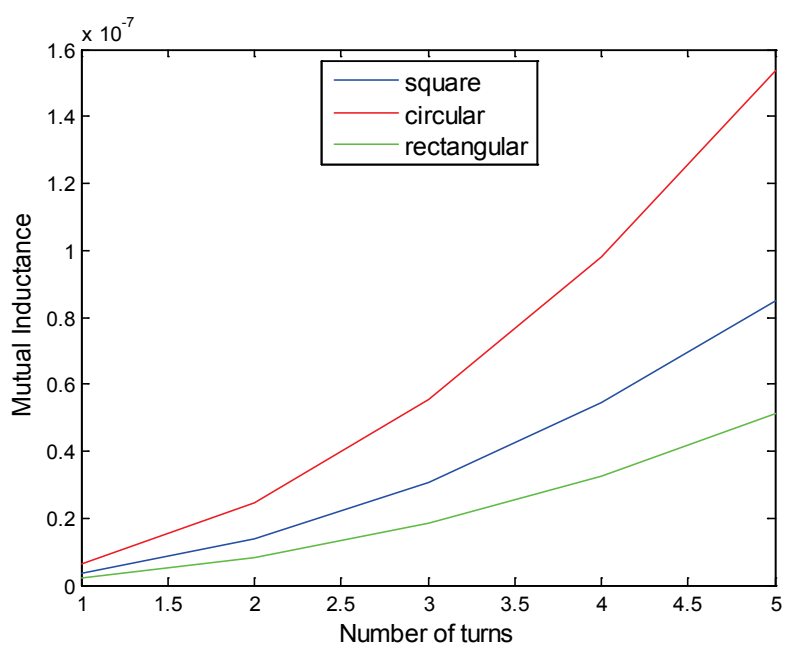

Figure $\mathbf{5}$ Measurement of mutal inductance as a function of coil turns

\subsection{Quality Factor Test}

The quality factor $Q$ of an inductive coil is the measure of the ratio of reactive components to its resistance. The quality factor indicates the ability of the coil to produce a better magnetic field. The quality factor of a coil can be expressed as,

$Q=\frac{2 \times \pi \times f \times L}{R}$

Higher quality factor indicates the better performance of inductive coils, which are well suitable for the WPT applications.

The simulation experiment is conducted to understand the behaviour of the quality factor with a frequency of $150 \mathrm{kHz}$. The values of quality factor are measured by varying the number of turns of each coil and its corresponding values are recorded for the analysis. The quality factor of each coil is plotted as shown in Fig. 6. It is observed from the comparative plots that the quality factor of all three shapes is exactly the same if they are being operated at the same frequency. It also shows that the quality factor remains constant for a given material, if the length and volume of the coil remains the same. Hence the quality factor is not affected when the shape of 
the coil is changed and it is uniform for different shaped inductive coils, with its own value of inductance.

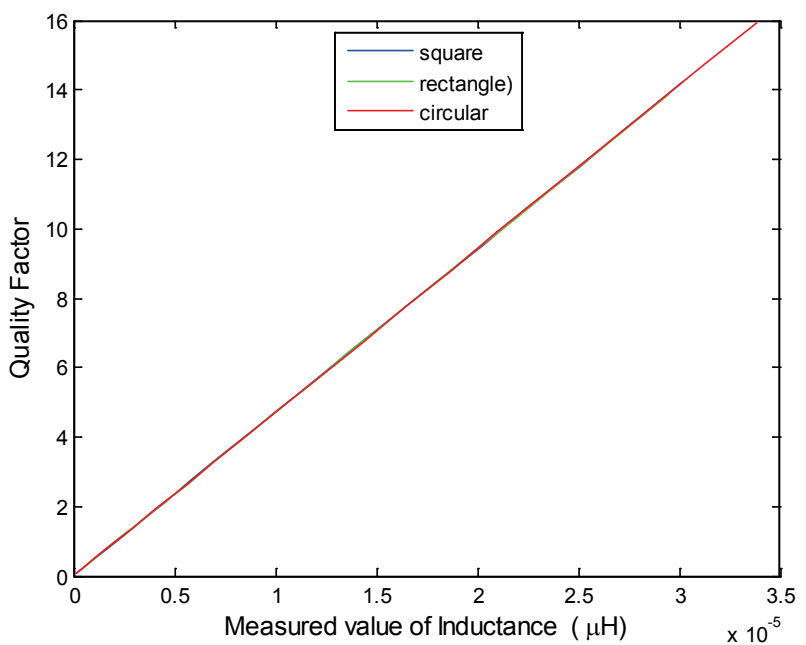

Figure 6 Measurement of quality factor as a function of inductance

\subsection{Magnetic Field Test}

As per the Faraday's law of electromagnetic induction, current carrying coils produce the magnetic field and then the conductor placed in the field zone will experience an induced e.m.f., which is directly proportional to the magnetic field strength. Hence the coil with higher magnetic field will produce better performance of WPT system. According to Biot-Savart law the magnetic intensity at a point due to a current element in a conductor is directly proportional to the current in that conductor and inversely proportional to the distance from the point to the wire.

The magnetic field of circular coil $B_{C}$ can be expressed with coil radius $r$, current I flowing through the coil, and $N$ number of turns as [8],

$$
B_{C}=\frac{\mu_{0} I N r^{2}}{2\left(z^{2}+r^{2}\right)^{\frac{3}{2}}}
$$

where $z$ is the distance from the centre on axis of coil symmetry to a measured point.

Similarly, the magnetic field of rectangular $B_{R}$ and square $B_{S}$ coils can be expressed as,

$$
\begin{aligned}
& B_{R}=\frac{2 \mu_{0} I N}{\pi} \times \\
& \times\left[\frac{a^{2}}{\left(4 z^{2}+a^{2}\right)\left(4 z^{2}+2 a^{2}\right)^{\frac{1}{2}}}+\frac{b^{2}}{\left(4 z^{2}+b^{2}\right)\left(4 z^{2}+2 b^{2}\right)^{\frac{1}{2}}}\right] \\
& B_{S}=\frac{4 \mu_{0} I N}{\pi}\left[\frac{a^{2}}{\left(4 z^{2}+a^{2}\right)\left(4 z^{2}+2 a^{2}\right)^{\frac{1}{2}}}\right]
\end{aligned}
$$

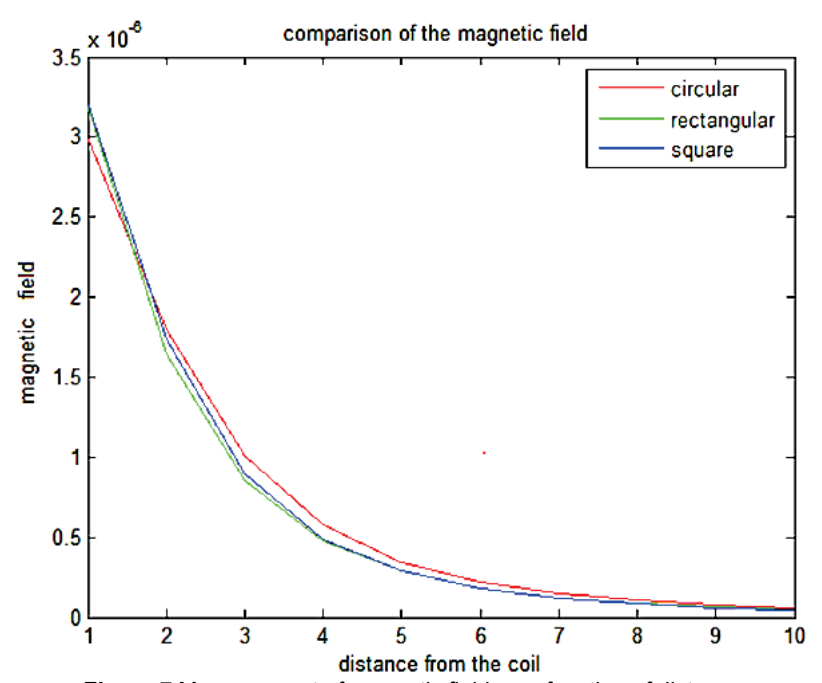

Figure 7 Measurement of magnetic field as a function of distance

The simulation is done to measure the magnetic field by considering a copper wire of $16 \mathrm{~cm}$ wounded into three turns. The point at which the magnetic field is to be measured was increased and its corresponding values are noted to analyse the magnetic field of three coils. The magnetic field gradually reduces as the point gets away from the coil. Comparing the performance of the coils by increasing the distance, it can be observed that the magnetic field linearly falls as the distance increases but at a given distance circular has the highest magnetic field. Moreover, the square and rectangular coils have the same magnetic property at a larger distance.
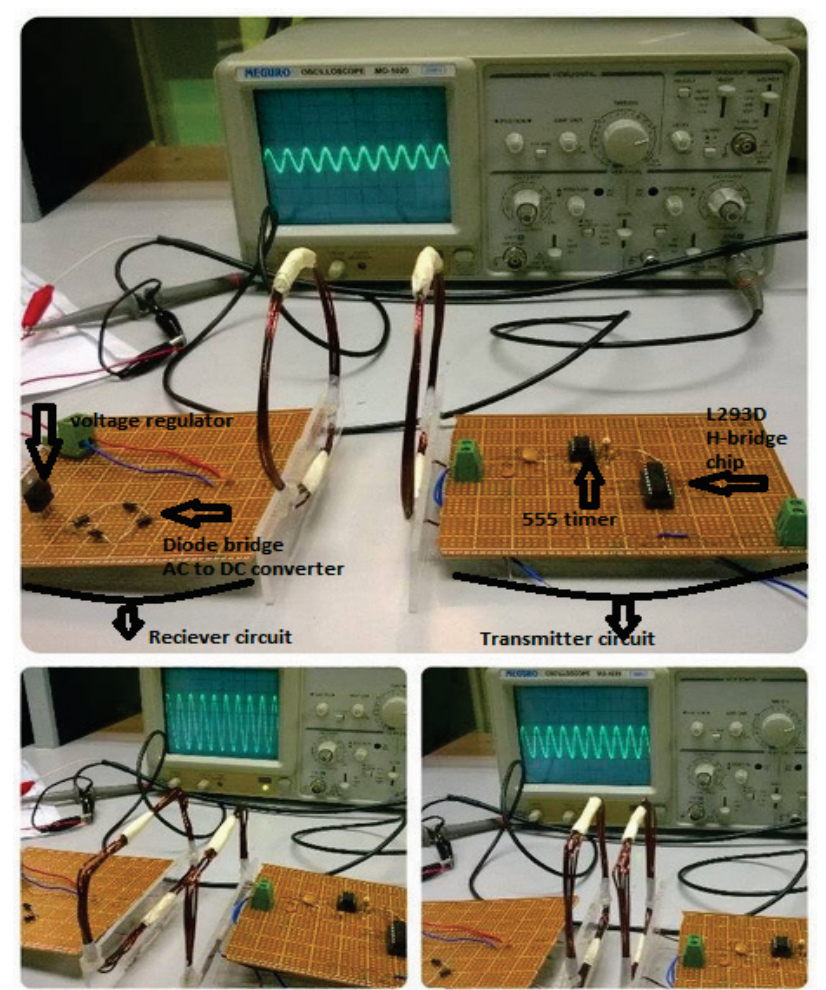

Figure 8 Experimental setup of three different coils used for efficiency measurement

\subsection{Efficiency Test}

Fig. 8 shows the experimental configuration of three diferent coils for efficiency measurement practically. An 
appropriate transmitter and reciever circuits are constructed with source and load coils to arrange the measurement setup. The output voltage is measured by applying $10 \mathrm{~V}$ input to these three configurations and then efficiency is calculated through the measurement of output voltage. The experimental setup as shown in Fig. 3 is used to conduct the efficiency measurement practically. All the theoritical parameters are analysed through the simulation work except efficiency. In order to confirm the theoritical parameters, the practical test bench was devoloped to measure the output voltage and then calculated the efficiency. In all three configurations, an $\mathrm{AC}$ input voltage of $10 \mathrm{~V}$ peak to peak was supplied to the source coil connected in the transmitter unit through frequency generator.

There are two different tests such as efficiency as a function of distance and frequency that are performed to analyse the power transfer efficiency. The frequency analysis is done by fixing the distance between the source and load coils as $3 \mathrm{~cm}$ as well as the distance analysis also conducted with the fixed value of resonant frequency of $150 \mathrm{kHz}$. The output voltage at the load coil is recorded with respect to the input frequency and distance.

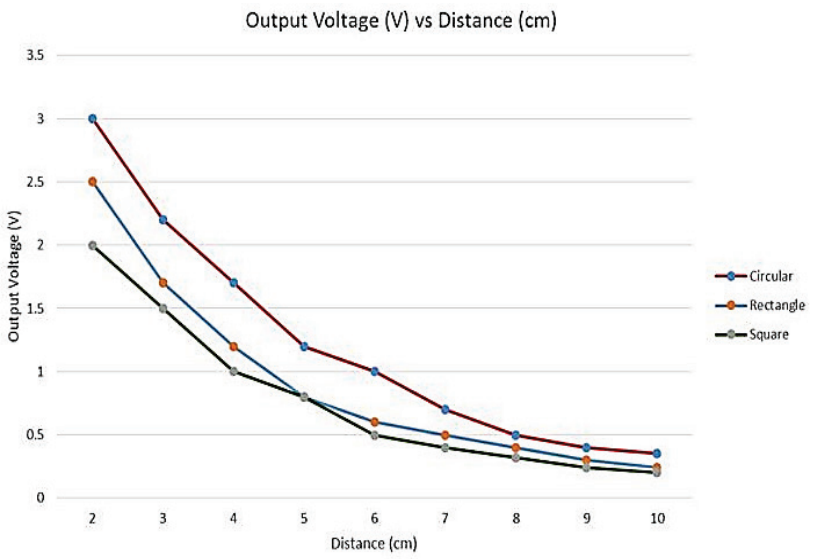

Figure 9 Measurement of output voltage with respect to coil distance for efficiency

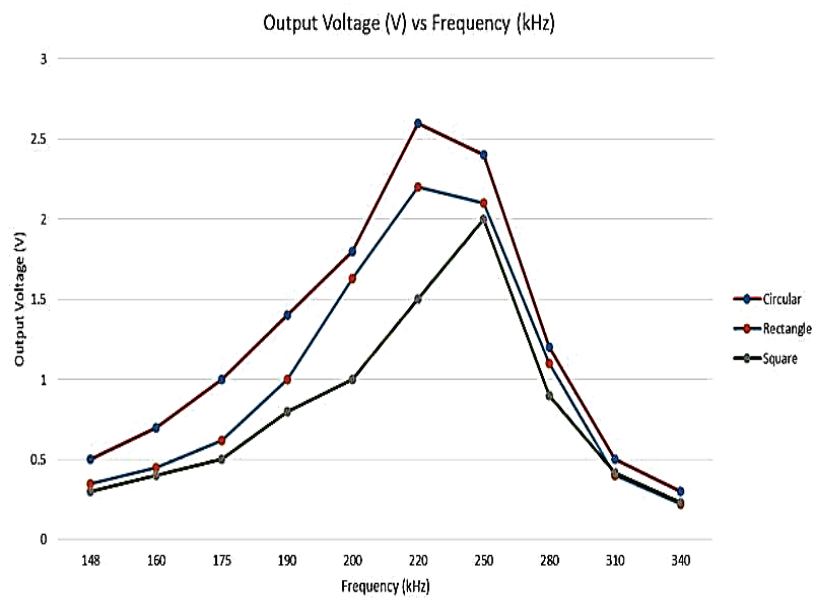

Figure 10 Resonant frequency analysis of three differnt coils for power transfer efficiency

The voltage at the load coil as a function of coil separation distance is illustrated in Fig. 9. It is observed that the output voltage exponentially drops as the distance between the coils is increased. The inductive coils have stronger magnetic field in the near field zone and weaker in the far field zone of the coils. The behaviour of all three coils is uniform and reduces when the distance of coil separation is increased. As similar, the analysis of efficiency with respect to the resonant frequency is also performed and its analysis is illustrated in Fig. 10. Under the resonance frequency, all the coils are producing maximum response, then decreasing to left and right of this frequency. The resonant frequency produced by circular and rectangular coils is almost the same but it is slightly deviated for the square coil. It is also observed that the circular coil produces higher voltage under the resonant frequency than the other two coils. Therefore it can be concluded that the circular coil produces better power transfer efficiency in both the cases as compared with rectangular and square shaped coils.

\section{CONCLUSION}

The performance analysis of inductive coils using their shape for wireless power transfer applications has been evaluated. Three different coils such as circular, rectangular, and square were designed and simulated to understand the electromagnetic features of inductive coils. The parameters such as self and mutual inductance, coupling coefficient, quality factor, magnetic field and efficiency were analysed using the Maxwell simulation platform in order to evaluate the coil parameters. After that, the power transfer efficiency is analysed through the laboratory experiments. This was done via using the practically wounded three lab scale coils and their output voltage measurements. It is observed that the circular coil has better electromagnetic features in all the aspects compared to the other two shapes. A practical resonance frequency analysis is also presented to ensure the power transfer efficiency of the coil configurations. The significant matter among all the coil shapes is that power transfer efficiency shoots up to the maximum when source and load coils are placed in a very close distance and decreases accordingly. The results confirm that the circular (29\%) combination possesses better efficiency as compared to rectangular $(25 \%)$ and square $(23 \%)$ shapes. The outcomes of this work provide the coil suitability analysis for wireless power transfer applications.

\section{REFERENCES}

[1] Tesla, N. (1914). U. S. Patent 1,119,732

[2] Kurs, A. et al. (2007). Wireless power transfer via strongly coupled magnetic resonances. Science Magazine, 317(5834), 83-86. https://doi.org/10.1126/science.1143254

[3] Karalis, A., Joannopoulos, J. D., \& Soljacic, M. (2008). Efficient wireless non-radiative mid-range energy transfer. Annals of Physics, 323, 34-48. https://doi.org/10.1016/j.aop.2007.04.017

[4] Arsad, A. et al. (2016). An Activity Monitoring System for Senior Citizens Living Independently Using Capacitive Sensing Technique. 2016 IEEE Int. Instrum. Meas. Technol. Conf., 1, 449-454.

[5] Sample, A. P., Meyer, A., \& Smith. J. R. (2010). Analysis, experimental results, and range adaption of magnetically coupled resonators for wireless power transfer. IEEE Transactions on Industrial Electronics, 58(2), 544-554. https://doi.org/10.1109/TIE.2010.2046002 
[6] Nagoorkar, V. (2012). Mid-range magnetically-coupled resonant circuit wireless power transfer. $\mathrm{PhD}$ thesis, the University of Texas at Tyler, May 2012.

[7] Hoang, H. \& Bien, F. (2012). Maximizing efficiency of electromagnetic resonance wireless power transmission systems with adaptive circuits. Wireless Power Transfer Principles and Engineering Explorations, 207-226. https://doi.org/10.5772/28922

[8] Wheeler, H. A. (1928) Simple inductance formulas for radio coils. Proc. of the Institute of Radio Engineers, 16, 1398-1400. https://doi.org/10.1109/JRPROC.1928.221309

[9] Hirayama, H. (2012). Equivalent circuit and calculation of its parameters of magnetic coupled resonant wireless power transfer. Wireless Power Transfer Principles and Engineering Explorations, 117-132. https://doi.org/10.5772/28806

[10] Bondar, H., Oree, S., Jagoo, Z., Ichikawa, K. (2013). Estimate of the maximum range achievable by nonradiating wireless power transfer or near-field communication systems. Journal of Electrostatics, 71(4), 648-655. https://doi.org/10.1016/j.elstat.2013.03.004

[11] Dionigi, M., Costanzo, A., \& Mongiardo, M. (2012). Network methods for analysis and design of resonant wireless power transfer systems. Wireless Power Transfer Principles and Engineering Explorations, 65-94. https://doi.org/10.5772/25281

[12] Hwang, H., Lee, B., Moon, J., Park, S., Jeong, C.-H., \& Kim, S.-W. (2014). Investigation of wireless power transfer in multi-coil environment. Proc. IEEE International Conference on Consumer Electronics / Las Vegas, USA, 315-316. https://doi.org/10.1109/ICCE.2014.6776019

[13] Nataraj, C., Khan, S., Habaebi, M. H., \& Muthalif, A. G. A. (2016). Resonant Coils Analysis for Inductively Coupled Wireless Power Transfer Applications. 2016 IEEE Int. Instrum. Meas. Technol. Conf., 1, 109-114. https://doi.org/10.1109//2MTC.2016.7520343

[14] Conway, J. T. (2007). Inductance calculations for noncoaxial coils using Bessel functions. IEEE Transactions on magnetics, 43(3), 1023-1034. https://doi.org/10.1109/TMAG.2006.888565

[15] Villa, J. L., Sallán, J., Llombart, A., \& Sanz, J. F. (2009). Design of a high frequency Inductively Coupled Power Transfer system for electric vehicle battery charge. Applied Energy, 86(3), 355-363.

https://doi.org/10.1016/j.apenergy.2008.05.009

\section{Contact information:}

Chandrasekharan NATARAJ, Senior Lecturer

Corresponding author

Asia Pacific University of Technology and Innovation

Lot 6, Technology Park Malaysia, Bukit Jalil,

57000 Kuala Lumpur, Malaysia

chander@apu.edu.my

Dr. Sheroz KHAN, Associate Professor

International Islamic University Malaysia (IIUM),

Jalan Gombak, 53100 Kuala Lumpur,

Selangor, Malaysia

sheroz@iium.edu.my

Dr. Mohammed Hadi HABAEBI, Associate Professor

International Islamic University Malaysia (IIUM),

Jalan Gombak, 53100 Kuala Lumpur,

Selangor, Malaysia

habaebi@ilum.edu.my

Dr. Asan G.A. MUTHALIF, Associate Professor International Islamic University Malaysia (IIUM), Jalan Gombak, 53100 Kuala Lumpur,

Selangor, Malaysia

asan@iium.edu.my 